Journal for

ImmunoTherapy of Cancer

\title{
Combination checkpoint blockade for metastatic cutaneous malignancies in kidney transplant recipients
}

\author{
Megan H Trager (D) , ${ }^{1}$ Shana M Coley (D) , ${ }^{2}$ Geoffrey Dube, ${ }^{3}$ Shaheer Khan, ${ }^{4}$ \\ Matthew Ingham, ${ }^{4}$ Faramarz H Samie, ${ }^{1}$ Larisa J Geskin, ${ }^{1}$ Diana McDonnell, ${ }^{4}$ \\ Daniel Brouder, ${ }^{5}$ Yvonne Saenger, ${ }^{4}$ Richard Carvajal ${ }^{4}$
}

To cite: Trager MH, Coley SM, Dube G, et al. Combination checkpoint blockade for metastatic cutaneous malignancies in kidney transplant recipients. Journal for ImmunoTherapy of Cancer 2020;8:e000908. doi:10.1136/ jitc-2020-000908

- Additional material is published online only. To view please visit the journal online (http://dx.doi.org/10.1136/jitc2020-000908).

Accepted 05 May 2020

\section{Check for updates}

(c) Author(s) (or their employer(s)) 2020. Re-use permitted under CC BY-NC. No commercial re-use. See rights and permissions. Published by BMJ.

${ }^{1}$ Department of Dermatology, Columbia University Irving Medical Center, New York, New York, USA

${ }^{2}$ Department of Pathology and Cell Biology, Division of Renal Pathology, Columbia University Irving Medical Center, New York, New York, USA

${ }^{3}$ Department of Medicine,

Division of Nephrology, Columbia University Irving Medical Center, New York, New York, USA

${ }^{4}$ Department of Medicine, Division of Hematology/ Oncology, Columbia University Irving Medical Center, New York, New York, USA

${ }^{5}$ Ocean Renal Associates, Brick, New Jersey, USA

Correspondence to

Dr Richard Carvajal;

rdc2150@cumc.columbia.edu

\section{ABSTRACT}

Background Immune checkpoint blockade has emerged as a highly effective treatment for patients with metastatic melanoma and cutaneous squamous cell carcinoma. Nivolumab blocks the interactions between programmed cell death protein 1 and programmed death ligand 1 allowing for activation of a latent immune response against the malignancy. Ipilimumab binds to and blocks cytotoxic T-lymphocyte-associated protein 4, alleviating the negative regulation of $\mathrm{T}$-cell activation that is mediated by that checkpoint. Combination therapy with nivolumab and ipilimumab is associated with longer overall survival at 5 years compared with nivolumab monotherapy. Solid organ transplant recipients have a significantly higher risk of malignancies compared with the general population. There is limited data surrounding the efficacy of combination immunotherapy in solid organ transplant recipients, as these patients were excluded from seminal trials due to risk of organ rejection.

Case presentations Here we present four cases of combination immunotherapy in kidney transplant recipients. Three patients had metastatic melanoma, and one patient had metastatic cutaneous squamous cell carcinoma. Two patients had radiographic responses from immunotherapy, one patient had stable disease, and one patient had disease progression. Only one patient had biopsy-proven rejection. At last follow-up, three patients had functioning grafts, though one required hemodialysis after treatment, and one patient succumbed to disease, but graft function remained intact throughout her course. Conclusions These cases describe the use of ipilimumab and nivolumab combination immunotherapy for cutaneous malignancies in kidney transplant recipients. They highlight the potential to preserve kidney graft function while effectively treating the disease.

\section{BACKGROUND}

Immune checkpoint blockade has emerged as a standard treatment for melanoma, ${ }^{1-5}$ cutaneous squamous cell carcinoma (cSCC), and others. ${ }^{7}$ Ipilimumab binds cytotoxic T-lymphocyte-associated protein 4 (CTLA-4), preventing normal ligand binding, thereby alleviating negative regulation of T-cell activation. Nivolumab, pembrolizumab, and cemiplimab interfere with a separate T-cell negative regulation pathway, by blocking the interactions between programmed cell death protein 1 (PD-1) on exhausted effector $\mathrm{T}$ cells and its ligands, PD-L1 and PD-L2. ${ }^{7}$ Blockade of CTLA-4 or PD-1/PD-L1 allows for activation of a latent immune response to cancer antigens, especially in highly immunogenic malignancies such as melanoma and cSCC. CheckMate 067 found greater 5-year survival in patients who received combination ipilimumab and nivolumab or nivolumab alone compared with ipilimumab alone $\left(52 \%, 44 \%\right.$, and $22 \%$, respectively). ${ }^{89}$ Currently, dual therapy is utilized in aggressive cases, although this has not been proven to improve survival. Higher power studies with longer follow-up may show a significant survival difference between combination ipilimumab and nivolumab versus nivolumab monotherapy.

Solid organ transplant recipients (SOTR) have increased rates of cancer, which is the second leading cause of death in this population. ${ }^{1011}$ This is attributed to long-term use of antirejection immunosuppressants causing impaired immune surveillance. SOTRs have a significantly higher incidence of $\mathrm{CSCC}^{12}$ (65fold to 250-fold increased risk) and malignant melanoma ${ }^{13}$ (two-fold to eight-fold increased risk). Immunosuppressed patients are particularly vulnerable to developing highly aggressive cSCC. In kidney SOTRs, cSCC accounts for over $70 \%$ of all new malignancies, affecting over $50 \%$ of kidney transplant patients. Post-transplant cSCC occurs earlier and is more aggressive than in non-transplant cohorts, with $30 \%$ of cSCC recurring within 1 year and up to $8 \%$ of disease associated with metastasis. ${ }^{14-16}$ Median survival after diagnosis of metastasis is 3 years. ${ }^{16} 17$

While multiple case reports and series of single agent checkpoint blockade in SOTRs exist, ${ }^{18}$ few cases treated with concurrent 
ipilimumab and anti-PD1 therapy have been reported. ${ }^{19-21}$ This patient exhibited partial response; however, graft rejection developed 21 days after treatment initiation. ${ }^{21}$ Here, we present four cases of metastatic cutaneous malignancy in the setting of kidney transplant treated with combination ipilimumab and nivolumab immunotherapy.

\section{CASE 1}

A 67-year-old Caucasian man with a history of membranous nephropathy diagnosed in 1997, status post two living donor kidney transplants, developed metastatic melanoma following over 10 years of immunosuppression (online supplementary table 1 ). The first kidney transplant (2008-2016) was pre-emptive from a living unrelated donor, with T-cell depletional induction (thymoglobulin) and maintenance immunosuppression with tacrolimus (2 $\mathrm{mg}$ twice daily), mycophenolic acid (360 $\mathrm{mg}$ twice daily), and prednisone ( $5 \mathrm{mg}$ four times a day). His first transplant course was complicated by invasive melanoma of the left scapular region in July 2015 (pT2a, N0), graft rejection treated with pulse steroids and intravenous immunoglobulin (IVIG), multiple invasive cutaneous SCCs and melanoma of the upper back in June 2016. The first graft failed due to chronic antibodymediated rejection in October 2016. He underwent repeat kidney transplantation in November 2016 from his daughter, with non-depletional induction (basiliximab), and in July 2019 was diagnosed with metastatic melanoma following left axillary lymph node biopsy.
Computed tomography (CT) and magnetic resonance imaging (MRI) showed liver, lung, and possible brain metastases (figure 1A). He was transitioned from tacrolimus to sirolimus ( $2 \mathrm{mg}$ four times a day), and the sirolimus was ultimately discontinued in August 2019. Following comprehensive discussion of risk and benefits, the patient initiated standard dosing ipilimumab $3 \mathrm{mg} /$ $\mathrm{kg}$ and nivolumab $1 \mathrm{mg} / \mathrm{kg}$ later that month given rapid progression and possible brain metastases, receiving two doses total. He subsequently developed septic shock and multiple organ failure, requiring initiation of hemodialysis. Restaging scans showed decreased size of melanoma in the liver, lungs, and spleen (figure 1B). Retrospective review of the images performed before and after combined checkpoint blockade with tumor measurements revealed a $40 \%$ response by Response Evaluation Criteria in Solid Tumors (RECIST) V.1.1 criteria, ${ }^{22}$ consistent with a partial response to therapy. Mycophenolic acid was discontinued in 2017. Given the critical illness, he was transitioned to nivolumab monotherapy, initially $240 \mathrm{mg}$ every 2 weeks and transitioned later to $480 \mathrm{mg}$ every 4 weeks. Of note, the patient's kidney function improved with supportive care, and he was taken off dialysis after approximately 1 month. Serum creatinine prior to initiation of immunotherapy was $0.79 \mathrm{mg} / \mathrm{dL}$ and has trended to $2.41 \mathrm{mg} / \mathrm{dL}$ as of December 2019 (figure 2). He remains on nivolumab with ongoing radiographic response over 6 months after initiating immunotherapy.
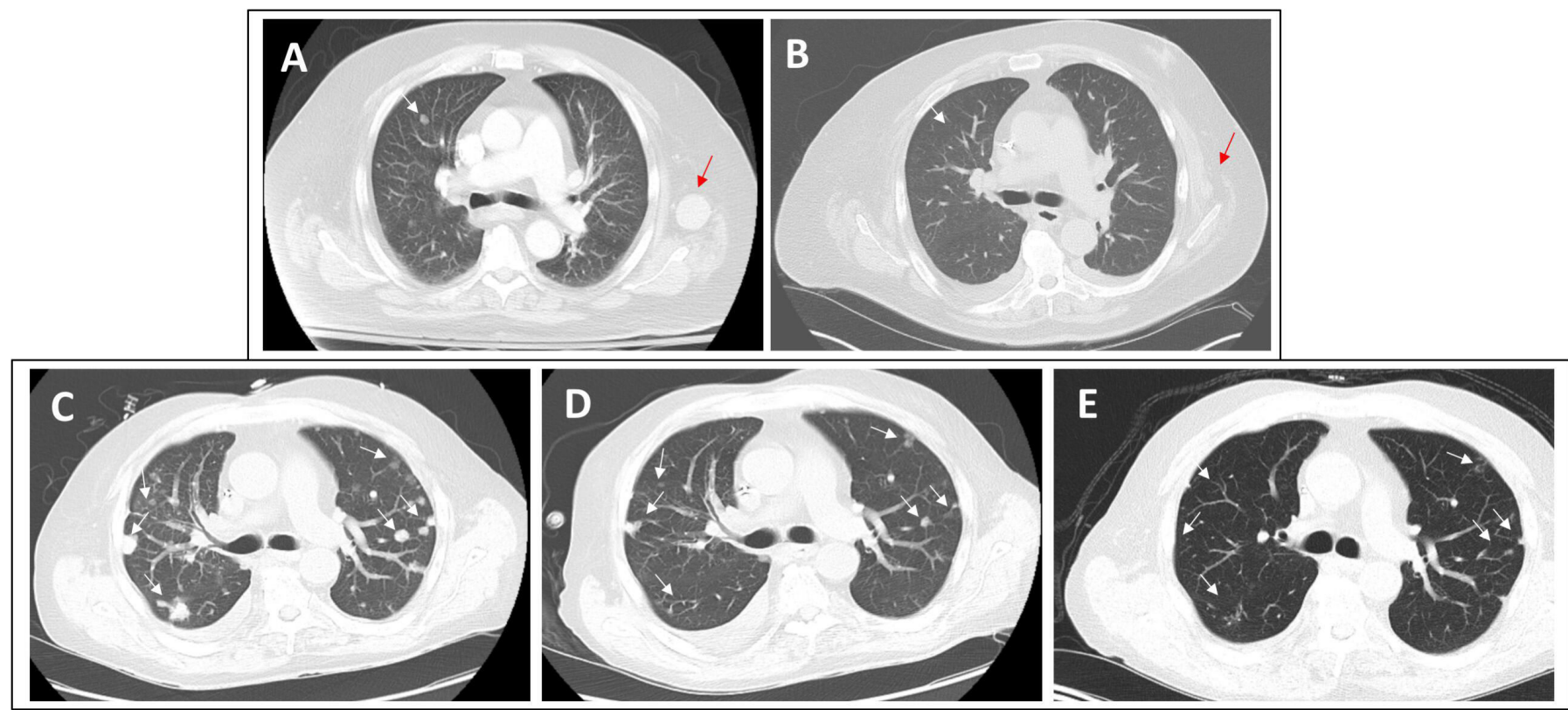

Figure 1 Radiographic improvement in disease. (A) Case 1: baseline chest CT in July 2019 at initiation of ipilimumab and nivolumab showing lung metastases; left axillary lesion (red arrow) was further examined with ultrasound and found to be a benign seroma or hematoma post left axillary biopsy. (B) CT in October 2019 after two doses of ipilimumab and nivolumab showing improvement in the lung metastases. (C) Case 3: baseline CT in August 2019 (at initiation of ipilimumab and nivolumab). (D) Case 3: October 2019 (after three treatments with ipilimumab and nivolumab). (E) Case 3: February 2019 (4 months after last dose ipilimumab). The CT scan shows significant reduction in lung metastases at the end of treatment with continual improvement 4 months after completing treatment with immunotherapy. Arrows track the metastases in each of the scans. 

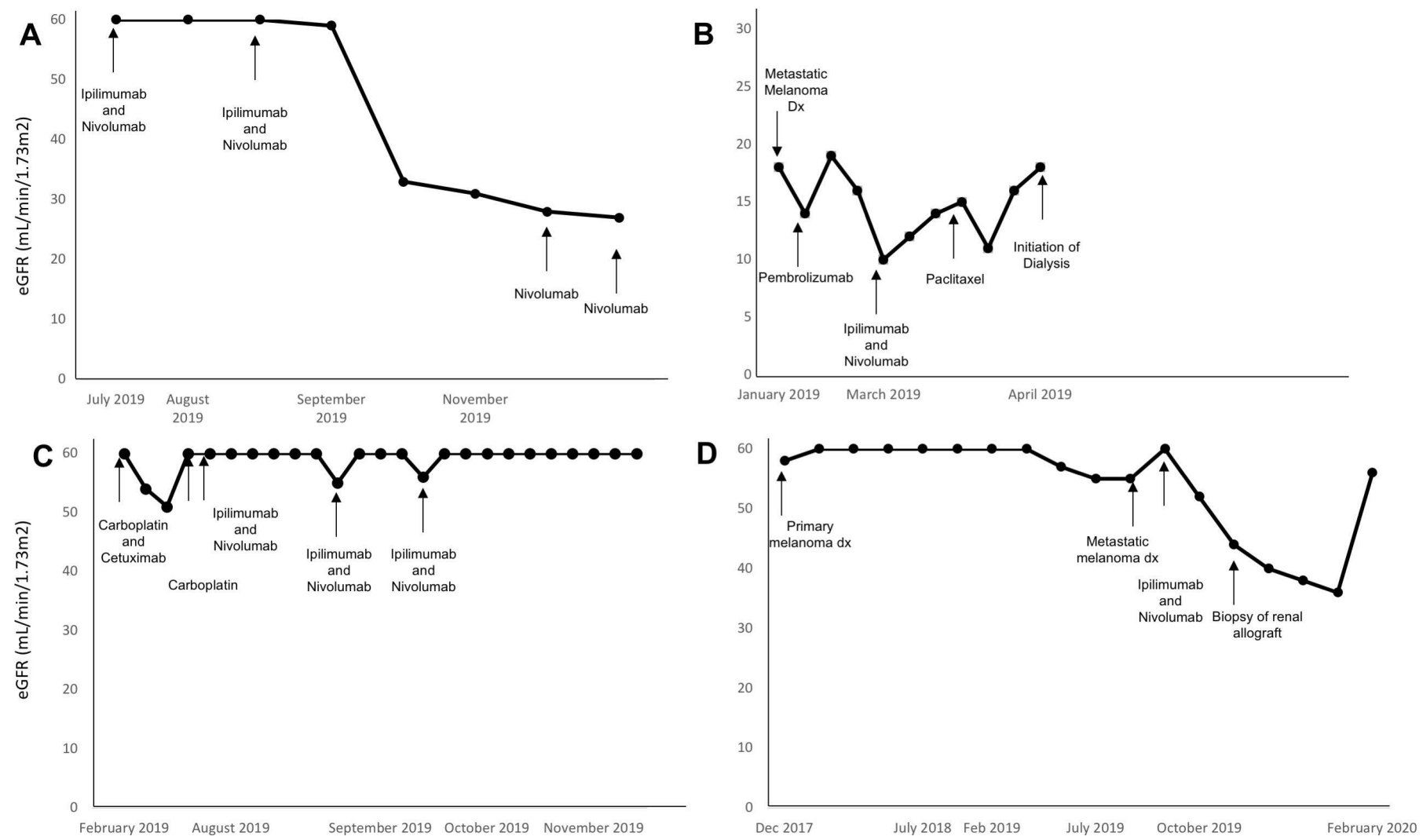

Figure 2 eGFR values over time after treatment with immunotherapy. Y label max is 60, indicates eGFR >60. (A) Case 1: metastatic melanoma diagnosed in July 2019. (B) Case 2: primary melanoma diagnosed in February 2018. (C) Case 3: metastatic cutaneous squamous cell carcinoma diagnosed in January 2019. (D) Case 4. primary melanoma diagnosed in December 2017. metastatic melanoma diagnosed in August 2019.

\section{CASE 2}

A Caucasian woman in her early 40s underwent preemptive living unrelated kidney transplantation (four antigen mismatch) in 2017 due to primary focal segmental glomerulosclerosis (FSGS), for which she had been treated with immunosuppressive regimens including cyclophosphamide (2004), steroids, tacrolimus, cyclosporine A, mycophenolic acid, rituximab, and IVIG, with biweekly plasmapheresis from 2014. T-cell depletional induction therapy was with thymoglobulin. Maintenance immunosuppression included tacrolimus ( $1 \mathrm{mg}$ four times a day), mycophenolic acid ( $360 \mathrm{mg}$ four times a day), and prednisone ( $5 \mathrm{mg}$ four times a day). Post-transplant, she received plasmapheresis and IVIG for biopsy-proven recurrent FSGS, though she never experienced biopsy-proven rejection. In February 2018, two synchronous primary non-ulcerated melanomas were diagnosed on her back $(0.8 \mathrm{~mm}, 1.5 \mathrm{~mm})$. She underwent wide local excision with $0.5 \mathrm{~mm}$ residual disease, no ulceration, and one mitosis $/ \mathrm{mm}^{2}$. Sentinel lymph node biopsy was negative. Due to close surgical margins, she completed adjuvant radiation therapy to the left back and shoulder. She discontinued treatment with mycophenolic acid around this time.

In December 2018, MRI showed a gluteal lesion, and biopsy in January 2019 confirmed recurrent melanoma (BRAF wildtype, NRAS mutant). Staging studies revealed two areas of distant soft tissue melanoma recurrence on the back and buttocks, from different lymphatic beds. Following discussion between the patient's oncologist and transplant physician, tacrolimus and plasmapheresis were discontinued in January 2019, resulting in singleagent maintenance immunosuppression with prednisone ( $5 \mathrm{mg}$ four times a day). Given that surgery was felt unlikely to be curative, the patient received a single treatment with pembrolizumab $200 \mathrm{mg}$ IV in February 2019. A rash developed 1 week following treatment initiation, and at 1 month, reported increased growth in the nodules on the back and buttocks. CT imaging confirmed disease progression. She experienced worsening proteinuria and refractory edema related to recurrent FSGS and began dialysis. Because of rapid disease progression following a single dose of pembrolizumab, she was switched to treatment with the oncolytic virus vaccine, talimogene laherparepvec, and combination ipilimumab $3 \mathrm{mg} / \mathrm{kg}$ and nivolumab $1 \mathrm{mg} / \mathrm{kg}$ in March 2019. Following one course of combination therapy, she was admitted for seizures and altered mental status and found to have new spine, lung, and brain metastases. She received stereotactic radiosurgery for the brain metastasis. She received single agent chemotherapy with paclitaxel in April 2019 and combination paclitaxel/carboplatin in May 2019. The patient was subsequently admitted to the hospital with shortness of breath and found to have a large malignant 
pleural effusion. Subsequent scans showed further disease progression, and, given the patient's strong desire to continue on immunotherapy for melanoma, she was restarted on pembrolizumab $200 \mathrm{mg}$ IV q3 weeks in May 2019. Review of the images performed before and after combined checkpoint blockade by RECIST V.1.1 criteria revealed $28 \%$ progression in target lesions as well as the development of multiple new metastases following therapy, consistent with progression of disease. She ultimately passed away in June 2019.

\section{CASE 3}

A 58-year-old Caucasian man with a history of IgA nephropathy underwent kidney transplantation from deceased donors in 1996 and 2006. Maintenance regimen for the second kidney allograft consisted of tacrolimus $(0.5 \mathrm{mg}$ four times a day), sirolimus ( $1 \mathrm{mg}$ every other day), and mycophenolate mofetil (500 mg twice daily). His post-transplant course was complicated by multiple melanoma and non-melanoma skin cancers, ultimately developing metastatic cSCC in late 2018. Positron emission tomography-computed tomography (PET-CT) in December 2018 revealed bilateral fludeoxyglucose (FDG)-avid neck nodes, a large skin lesion at the base of the left posterior neck, and a hypermetabolic right lower lung nodule suspicious for metastatic disease. He was treated with carboplatin plus cetuximab in January 2019, complicated by severe nausea and vomiting requiring hospitalization. Transition to single agent cetuximab caused significant fatigue, so single agent carboplatin was trialed in early March 2019. Palliative radiation therapy was administered to the shoulder. Staging scans showed stable disease after the two cycles of carboplatin-based therapy. After discussion with his transplant nephrologist, he began cemiplimab $350 \mathrm{mg}$ IV every 3 weeks. He was continued on sirolimus ( $1 \mathrm{mg}$ three times weekly) with monitoring by his transplant nephrologist. He underwent five cycles of treatment with cemiplimab which he tolerated well. However, restaging scans showed disease progression, with growth of the dominant right middle lobe mass as well as many newer, smaller pulmonary nodules. Further options were discussed, and a plan was made to start combination immunotherapy with ipilimumab $3 \mathrm{mg} / \mathrm{kg}$ and nivolumab $1 \mathrm{mg} / \mathrm{kg}$ in late July 2019 . He took prednisone ( $5 \mathrm{mg}$ four times a day) as prescribed by his transplant nephrologist to prevent graft failure. ${ }^{23}$ After three cycles of ipilimumab and nivolumab between July and September 2019, there was radiographic improvement and no evidence of toxicity (figure 1C-E). Review of the images at baseline and after combined checkpoint blockade demonstrated a $40 \%$ response by RECIST V.1.1 criteria consistent with a partial response. However, he was hospitalized for adverse effects including rash from September to October. The rash improved with systemic steroids. He has since been on observation with no further active therapy, with continued radiographic response 9 months following initiation of combination checkpoint blockade and with preserved kidney function.

\section{CASE 4}

A Caucasian man in his late 50 s with a history of end-stage kidney disease secondary to hypertension underwent his third kidney transplant from a living unrelated donor in November 2015. Induction regimen included thymoglobulin, and initial maintenance immunosuppression was with prednisone, tacrolimus, and mycophenolic acid. Due to development of multiple cSCCs, tacrolimus was switched to sirolimus in March 2016, and maintenance immusuppression included prednisone ( $5 \mathrm{mg}$ four times a day), mycophenolic acid ( $360 \mathrm{mg}$ twice daily), and sirolimus (2 $\mathrm{mg}$ alternating with $1 \mathrm{mg}$ daily). In 2017, he was diagnosed with primary melanoma and underwent resection but developed metastatic disease of the cervical lymph nodes and liver in August 2019. Mycophenolic acid was discontinued in September 2019, and he continued prednisone ( $5 \mathrm{mg}$ four times a day) and sirolimus ( $1 \mathrm{mg}$ four times a day). He started combination immunotherapy (nivolumab and ipilimumab) in October 2019. Serum creatinine rose from a baseline of $1.1-1.3$ to $1.84 \mathrm{mg} / \mathrm{dL}$ in mid-November 2019 at which time a kidney allograft biopsy was performed. Biopsy showed moderate interstitial inflammation and severe lymphocytic tubulitis with diffuse C4d positivity, consistent with mixed acute T-cell mediated and antibody-mediated rejection (figure 3 ). He was treated with $500 \mathrm{mg}$ IV solumedrol daily for 3 days. No clinical evidence of progression at 2-month follow-up after initiation of combination checkpoint blockade; however, no postcheckpoint blockade imaging has been performed. His graft function has improved following treatment for immunotherapy-associated rejection, with serum creatinine decreasing from $1.84 \mathrm{mg} / \mathrm{dL}$ at the time of biopsy in November 2019 to $1.32 \mathrm{mg} / \mathrm{dL}$ in February 2020.

\section{DISCUSSION AND CONCLUSIONS}

Checkpoint inhibitor use in SOTRs has been challenging due to risks of graft rejection following the release of inhibitory T-cell signals. ${ }^{24}$ The efficacy and toxicity of these agents is less well studied in this cohort, as the seminal trials leading to the approval of checkpoint inhibitors excluded SOTRs. ${ }^{25}$ Prospective studies evaluating the safety and efficacy of immune checkpoint blockade in SOTRs with cancer are ongoing, and a clinical trial was launched evaluating the use of tacrolimus, ipilimumab and nivolumab in kidney transplant recipients with malignancy that might benefit from immune checkpoint blockade. The published experience is currently limited to case series and retrospective cohort studies. ${ }^{7821}$

Three systematic reviews have examined the use of immunotherapies for malignancy in SOTRs. ${ }^{7021}$ One review included 39 patients with kidney, hepatic, or cardiac allografts. The majority of patients had metastatic 

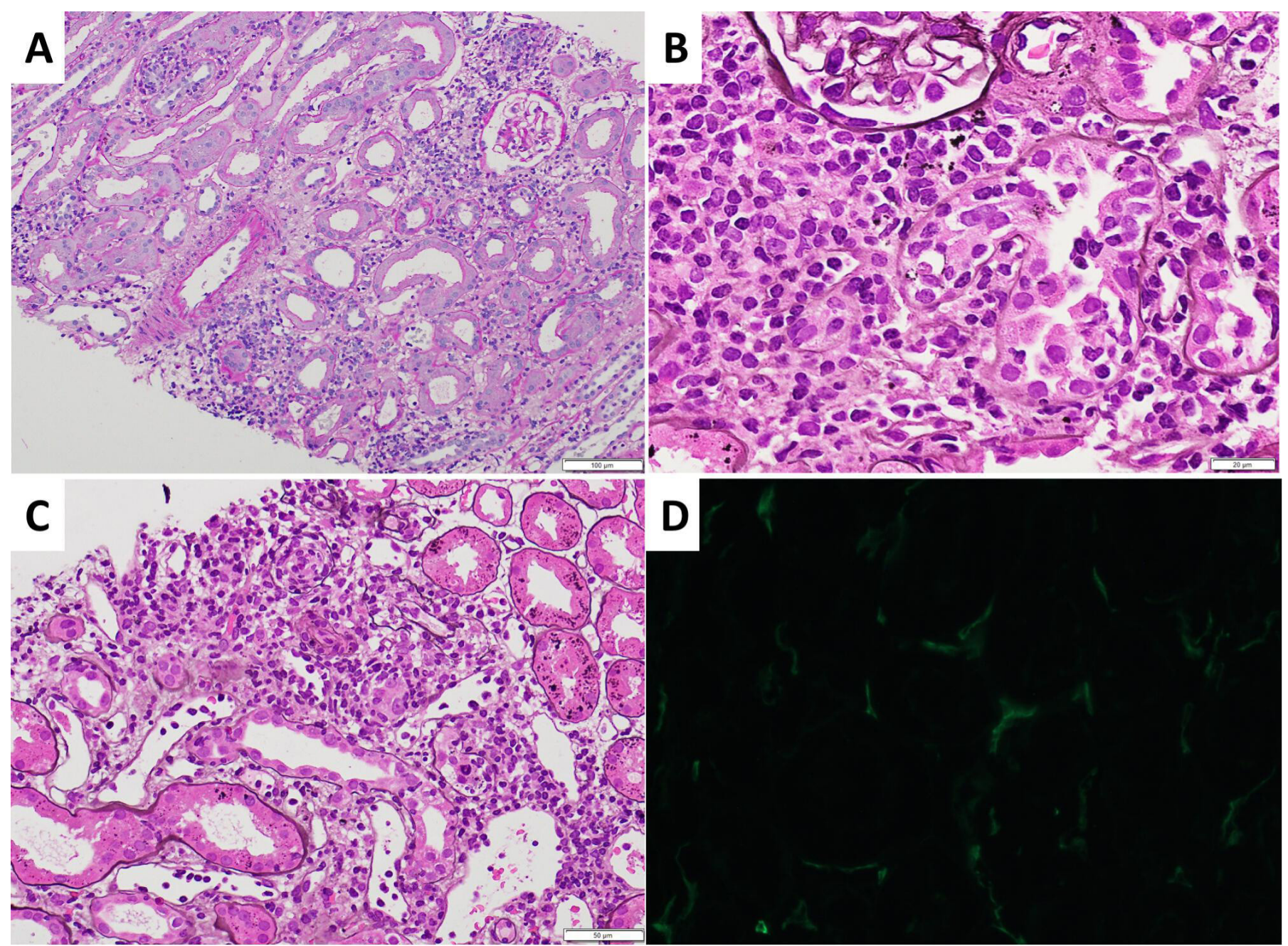

Figure 3 Kidney allograft biopsy in case 4, after treatment with combination immunotherapy and decreased immunosuppression, showing moderate interstitial inflammation, severe lymphocytic tubulitis, peritubular capillaritis and diffuse C4d positivity, consistent with mixed acute T-cell mediated and antibody-mediated rejection. (A) Periodic acid Schiff stain. (B and C) Jones methenamine silver stain. (D) C4d indirect immunofluorescence stain.

melanoma (62\%); 16/39 patients $(41 \%)$ had allograft rejection after initiation of checkpoint inhibitor therapy (48\% kidney, $36 \%$ hepatic, $20 \%$ cardiac) with no difference between those receiving anti-PD1 monotherapy, anti-CTLA-4 monotherapy, or combination treatment. The median time to organ rejection was 21 days after treatment initiation. ${ }^{26}$ Another systematic review of 57 SOTRs treated with ipilimumab, nivolumab, or pembrolizumab found that $37 \%$ of patients experienced organ rejection and $14 \%$ died due to graft failure. ${ }^{7}$ This study included 32 kidney, 20 liver, and five heart allograft recipients with rejection rates of $13 \%, 7 \%$, and $1 \%$, respectively. The authors concluded that physicians should exercise caution when treating this population with checkpoint inhibitors. However, the most common cause of death was malignancy $(64 \%)$ rather than graft rejection. In both reviews, the majority of patients received single agent immunotherapy with only one patient receiving combination therapy. More recently, a larger systematic review reported 83 SOTRs treated with immunotherapy (two-thirds anti-PD1/PDL1, 15.7\% anti-CTLA-4, and $10.8 \%$ combination therapy either sequentially or simultaneously). ${ }^{20}$ This included 53 kidney, 24 liver, and six heart transplants with similar rejection rates across organs and checkpoint inhibitors. Time since transplantation of at least 8 years was associated with lower risk of graft rejection, and history of prior allograft rejection was associated with higher risk. Patients on no other immunosuppression besides corticosteroids at initiation of checkpoint inhibitor therapy had a higher risk of rejection. At the end of the study, $19.3 \%$ of patients were alive without cancer progression or allograft rejection. Kidney transplant populations were the only group where there was similar mortality in patients with and without rejection. This shows that checkpoint inhibitor therapy may be a better option in these patients where hemodialysis is a life-saving alternative in case of rejection.

We present four cases of metastatic cutaneous malignancies in kidney transplant recipients treated with combination immunotherapy. One patient passed away from progressive malignant disease. Two of the four patients have shown durable radiographic improvement 
in disease after treatment. The fourth showed rejection on kidney allograft biopsy following immunotherapy and reduction in immunosuppression, with stabilized kidney function after high dose steroid treatment and no clinical evidence of disease progression although radiographic follow-up was not performed.

A recent paper found that combination immune checkpoint inhibitor therapy was associated with increased risk of immune-mediated acute kidney injury. ${ }^{27}$ In case 1 , transient hemodialysis was required for septic shock and multiple organ failure, though it was unclear whether or not these events were related to the immunotherapy, and the patient has shown improved kidney function on single-agent nivolumab therapy. Though hemodialysis was initiated in case 2, it bears repeating that this was due to recurrent FSGS-related graft dysfunction which developed prior to treatment with ipilimumab and nivolumab and not due to graft rejection. The third patient has shown preserved kidney allograft function and remains on immunotherapy. This further supports the use of immunotherapy in patients with kidney transplantation versus other SOTRs, as graft loss can be medically managed, while heart or lung transplantation would be much more difficult to address. ${ }^{7}$ Though each patient presented here was advised that graft loss was a highly probable outcome under immunotherapy, they elected to undergo treatment, as metastatic disease was of primary concern, and hemodialysis was an acceptable outcome.

Immunosuppressive regimens for prevention of kidney allograft rejection were comanaged with a kidney transplant physician in all cases. Previous studies have shown that frequency of allograft rejection varies based on the maintenance immunosuppressive regimen at the time of checkpoint inhibitor initiation. ${ }^{21}$ The systematic review from MD Anderson found that patients maintained on prednisone alone have higher risk of rejection $(78 \%)$ versus those continuing calcineurin inhibitor therapy $(11 \%)$, suggesting that aggressive reduction of maintenance immunosuppression increases rejection risk with checkpoint blockade. ${ }^{21}$ However, future studies are needed to determine the optimal immunosuppressive regimen to achieve a protective effect on graft function in the setting of blockade. Of our patients, one was initially treated with tacrolimus, then switched to sirolimus which was discontinued prior to checkpoint inhibitor therapy, leaving prednisone monotherapy for prevention of allograft rejection. The second patient was also treated with prednisone monotherapy for antirejection during immunotherapy, and the third and fourth patients continued treatment with sirolimus and prednisone during immune checkpoint blockade.

These cases add to the current literature describing ipilimumab and nivolumab combination immunotherapy for cutaneous malignancies in kidney transplant recipients. They highlight the possibility to preserve kidney graft function while improving outcomes from malignancy with combination immunotherapy.
Correction notice This article has been corrected since it was published Online First to remove the incorrect trial registration number from abstract that was inserted by error.

Contributors All authors contributed to this manuscript. MHT wrote the manuscript and prepared tables and figures. SMC helped with conception and interpretation of the work. She edited the manuscript. GD, SK, and LJG helped with editing and conceptualization of the manuscript. DMcD helped with editing of the manuscript and obtaining clinical images for figures. YS contributed to conceptualization and design of the manuscript as well as editing. RC conceptualized the project, helped with writing of the initial manuscript, making figures and tables, and editing the final draft.

Funding The authors have not declared a specific grant for this research from any funding agency in the public, commercial, or not-for-profit sectors.

Competing interests $\mathrm{RC}$ is a consultant for Astra Zeneca, BMS, Castle Biosciences, Foundation Medicine, Immunocore, Incyte, Merck, Novartis, Roche, Compugen, I-Mab, PureTech Health, Sanofi Genzyme, and Sorrento Therapeutics. $\mathrm{He}$ is on advisory boards for Aura Biosciences, Chimeron, and Rgenix.

Patient consent for publication Not required.

Provenance and peer review Not commissioned; externally peer reviewed.

Data availability statement The authors confirm that the data supporting the findings of this study are available in the manuscript and supplementary table.

Open access This is an open access article distributed in accordance with the Creative Commons Attribution Non Commercial (CC BY-NC 4.0) license, which permits others to distribute, remix, adapt, build upon this work non-commercially, and license their derivative works on different terms, provided the original work is properly cited, appropriate credit is given, any changes made indicated, and the use is non-commercial. See http://creativecommons.org/licenses/by-nc/4.0/.

\section{ORCID iDs}

Megan H Trager http://orcid.org/0000-0002-7330-1627

Shana M Coley http://orcid.org/0000-0002-2152-5469

\section{REFERENCES}

1 Eggermont AMM, Chiarion-Sileni V, Grob J-J, et al. Prolonged survival in stage III melanoma with ipilimumab adjuvant therapy. $N$ Engl J Med 2016;375:1845-55.

2 Larkin J, Chiarion-Sileni V, Gonzalez R, et al. Combined nivolumab and ipilimumab or monotherapy in untreated melanoma. $N$ Engl J Med 2015;373:23-34.

3 Weber J, Mandala M, Del Vecchio M, et al. Adjuvant nivolumab versus ipilimumab in resected stage III or IV melanoma. $N$ Engl J Med 2017;377:1824-35.

4 Larkin J, Hodi FS, Wolchok JD. Combined nivolumab and ipilimumab or monotherapy in untreated melanoma. $N$ Engl J Med 2015;373:23-34.

5 Eggermont AMM, Blank CU, Mandala M, et al. Adjuvant pembrolizumab versus placebo in resected stage III melanoma. $N$ Engl J Med 2018;378:1789-801.

6 Migden MR, Rischin D, Schmults CD, et al. Pd-1 blockade with Cemiplimab in advanced cutaneous squamous-cell carcinoma. $N$ Engl J Med 2018;379:341-51.

7 Fisher J, Zeitouni N, Fan W, et al. Immune checkpoint inhibitor therapy in solid organ transplant recipients: a patient-centered systematic review. J Am Acad Dermatol 2019:S0190-9622(19)323175.

8 Wolchok JD, Chiarion-Sileni V, Gonzalez R, et al. Overall survival with combined nivolumab and ipilimumab in advanced melanoma. $N$ Engl J Med 2017;377:1345-56.

9 Larkin J, Chiarion-Sileni V, Gonzalez R, et al. Five-Year survival with combined nivolumab and ipilimumab in advanced melanoma. $N$ Engl J Med 2019;381:1535-46.

10 Engels EA, Pfeiffer RM, Fraumeni JF, et al. Spectrum of cancer risk among US solid organ transplant recipients. JAMA 2011;306:1891-901.

11 Acuna SA, Fernandes KA, Daly C, et al. Cancer mortality among recipients of solid-organ transplantation in Ontario, Canada. JAMA Oncol 2016;2:463-9.

12 Krynitz B, Olsson H, Lundh Rozell B, et al. Risk of basal cell carcinoma in Swedish organ transplant recipients: a populationbased study. Br J Dermatol 2016;174:95-103.

13 Fattouh K, Ducroux E, Decullier E, et al. Increasing incidence of melanoma after solid organ transplantation: a retrospective epidemiological study. Transpl Int 2017;30:1172-80. 
14 Harwood CA, Mesher D, McGregor JM, et al. A surveillance model for skin cancer in organ transplant recipients: a 22-year prospective study in an ethnically diverse population. Am J Transplant 2013;13:119-29.

15 Buell JF, Hanaway MJ, Thomas M, et al. Skin cancer following transplantation: the Israel Penn international transplant tumor registry experience. Transplant Proc 2005;37:962-3.

16 Wisgerhof HC, van der Geest LGM, de Fijter JW, et al. Incidence of cancer in kidney-transplant recipients: a long-term cohort study in a single center. Cancer Epidemiol 2011;35:105-11.

17 Martinez J-C, Otley CC, Stasko T, et al. Defining the clinical course of metastatic skin cancer in organ transplant recipients: a multicenter collaborative study. Arch Dermatol 2003;139:301-6.

18 Chae YK, Galvez C, Anker JF, et al. Cancer immunotherapy in a neglected population: the current use and future of T-cell-mediated checkpoint inhibitors in organ transplant patients. Cancer Treat Rev 2018;63:116-21.

19 Miller DM, Faulkner-Jones BE, Stone JR, et al. Complete pathologic response of metastatic cutaneous squamous cell carcinoma and allograft rejection after treatment with combination immune checkpoint blockade. JAAD Case Rep 2017;3:412-5.
20 d'Izarny-Gargas T, Durrbach A, Zaidan M. Efficacy and tolerance of immune checkpoint inhibitors in transplant patients with cancer: a systematic review. Am J Transplant 2020:15811.

21 Abdel-Wahab N, Safa H, Abudayyeh A, et al. Checkpoint inhibitor therapy for cancer in solid organ transplantation recipients: an institutional experience and a systematic review of the literature. $J$ Immunother Cancer 2019;7:106.

22 Eisenhauer EA, Therasse P, Bogaerts J, et al. New response evaluation criteria in solid tumours: revised RECIST guideline (version 1.1). Eur $J$ Cancer 2009;45:228-47.

23 Barnett R, Barta VS, Jhaveri KD. Preserved renal-allograft function and the PD-1 pathway inhibitor nivolumab. $N$ Engl J Med 2017;376:191-2.

24 Smedman TM, Line P-D, Guren TK, et al. Graft rejection after immune checkpoint inhibitor therapy in solid organ transplant recipients. Acta Oncol 2018;57:1414-8.

25 Kittai AS, Oldham H, Cetnar J, et al. Immune checkpoint inhibitors in organ transplant patients. J Immunother 2017;40:277-81.

26 Perazella MA, Shirali AC. Immune checkpoint inhibitor nephrotoxicity: what do we know and what should we do? Kidney Int 2020;97:62-74.

27 Cortazar FB, Kibbelaar ZA, Glezerman IG, et al. Clinical features and outcomes of immune checkpoint inhibitor-associated AKI: a multicenter study. J Am Soc Nephrol 2020;31:435-46. 


\section{Correction: Combination checkpoint blockade for metastatic} cutaneous malignancies in kidney transplant recipients

Trager MH, Coley SM, Dube G, et al. Combination checkpoint blockade for metastatic cutaneous malignancies in kidney transplant recipients. J Immunother Cancer 2020;8:e000908. doi: 10.1136/jitc-2020-000908

This article has been corrected since it was first published to remove the incorrect trial registration number which was erroneously inserted in the abstract.

(C) Author(s) (or their employer(s)) 2021. No commercial re-use. See rights and permissions. Published by BMJ.

J Immunother Cancer 2021;9:e000908corr1. doi:10.1136/jitc-2020-000908corr1

A) Check for updates 